\title{
Integration of genetic, genomic and transcriptomic information identifies putative regulators of adventitious root formation in Populus
}

Cintia L. Ribeiro ${ }^{1,2,4 \dagger}$, Cynthia M. Silva ${ }^{1 \dagger}$, Derek R. Drost ${ }^{1,2,5}$, Evandro Novaes ${ }^{1,6}$, Carolina R. D. B. Novaes ${ }^{1,6}$, Christopher Dervinis ${ }^{1}$ and Matias Kirst ${ }^{1,2,3^{*}}$

\begin{abstract}
Background: Adventitious roots (AR) develop from tissues other than the primary root, in a process physiologically regulated by phytohormones. Adventitious roots provide structural support and contribute to water and nutrient absorption, and are critical for commercial vegetative propagation of several crops. Here we quantified the number of AR, root architectural traits and root biomass in cuttings from a pseudo-backcross population of Populus deltoides and Populus trichocarpa. Quantitative trait loci (QTL) mapping and whole-transcriptome analysis of individuals with alternative QTL alleles for AR number were used to identify putative regulators of AR development.

Results: Parental individuals and progeny showed extensive segregation for AR developmental traits. Quantitative trait loci for number of AR mapped consistently in the same interval of linkage group (LG) II and LG XIV, explaining $7-10 \%$ of the phenotypic variation. A time series transcriptome analysis identified 26,121 genes differentially expressed during AR development, particularly during the first $24 \mathrm{~h}$ after cuttings were harvested. Of those, 1929 genes were differentially regulated between individuals carrying alternative alleles for the two QTL for number of $A R$, in one or more time point. Eighty-one of these genes were physically located within the QTL intervals for number of AR, including putative homologs of the Arabidopsis genes SUPERROOT2 (SUR2) and TRYPTOPHAN SYNTHASE ALPHA CHAIN (TSA1), both of which are involved in the auxin indole-3-acetic acid (IAA) biosynthesis pathway.

Conclusions: This study suggests the involvement of two genes of the tryptophan-dependent auxin biosynthesis pathway, SUR2 and TSA 1, in the regulation of a critical trait for the clonal propagation of woody species. A possible model for this regulation is that poplar individuals that have poor AR formation synthesize auxin indole-3-acetic acid (IAA) primarily through the tryptophan (Trp) pathway. Much of the Trp pathway flux appears to be directed to the synthesis of indole glucosinolates (IG), as suggested by the over-expression of SUR2. Individuals that are efficient in AR formation may utilize alternative (non-Trp) pathways to synthesize IAA, based on the observation that they down-regulate the expression of TSA1, one of the critical steps in the synthesis of tryptophan.
\end{abstract}

Keywords: Adventitious root, QTL, Populus, SUR2, Vegetative propagation

\footnotetext{
* Correspondence: mkirst@ufl.edu

${ }^{\dagger}$ Equal contributors

'School of Forest Resources and Conservation, University of Florida, P.O. Box

110410, Gainesville, FL 32611, USA

${ }^{2}$ Plant Molecular and Cellular Biology Graduate Program, University of Florida,

P.O. Box 110690, Gainesville, FL 32611, USA

Full list of author information is available at the end of the article
} 


\section{Background}

Adventitious roots (AR) develop from plant tissues other than the primary root, providing structural support and contributing to water and nutrient absorption [1]. Adventitious and lateral roots follow a common developmental program, although dedifferentiation of already committed cells is required for AR formation [2]. Formation of AR occurs in three phases that may overlap: (1) dedifferentiation of previously committed cells (typically secondary phloem cells); (2) induction, when cells begin to divide to form an internal root meristem; and (3) elongation, when the root-primordia grows and emerges from the stem [1]. When ARs are developed from stem cuttings, wound response also occurs, which activates repair responses and systemic signaling cycles [3]. Usually, AR primordia arise close to the phloem and cambium, at the ray cells or in bud or leaf gaps. Adventitious roots may also arise in the pericycle, between the endodermis and phloem in roots [3]. The timing of each phase of AR formation varies among species and depends on external stimuli, but the first root meristems are frequently observed after $96 \mathrm{~h}[1,4,5]$.

Phytohormones are critical endogenous factors in AR formation, acting directly on cell division and growth, or indirectly, interacting with other molecules or phytohormones [6]. Auxin is the principal phytohormone that initiates rooting and is critical for the first phases of AR development [7], although inhibitory during elongation. Ethylene is likely to interact with auxin to control adventitious rooting in stems or stem cuttings [2], with some studies suggesting that auxin promotes dedifferentiation through stimulation of ethylene synthesis [8]. Although ethylene is promotive during the first phase of dedifferentiation, it is inhibitory during the induction phase [4]. Cytokinins have also been shown to impact AR formation [9], and interact with auxin to form the quiescent center [7]. Gibberellins appear to negatively impact the initial formation of ARs by interfering with the polar transport of auxin [10], while acting positively in their emergence and elongation [11]. Finally, strigolactones have also shown to impact AR formation [12, 13], although the contribution to the phenotype through interactions with other hormones remains to be uncovered. Regardless of the mechanisms of hormonal regulation, the initial development of ARs is primarily controlled by the availability of auxin and its proper localization, while most other hormones act as inhibitors or in combination with auxin.

The general role of phytohormones in AR formation is relatively well known, but few genes implicated in this developmental process have been identified. While the genes and molecular mechanisms that regulate $A R$ in woody perennial species are unknown, AR development is clearly under significant genetic control in woody species (for instance, see $[14,15])$. Species and hybrids in the Aigeiros and Tacamahaca sections of the genus Populus are among the taxa that are able to produce adventitious roots from cuttings, but considerable variation in the degree and vigor of rooting exists [16]. Therefore, poplar hybrids are particularly suitable for studying the genetic control of AR formation because there is extensive variation for the trait among species, and well-established genetic and genomic resources [17-20]. Most important, the DNA sequence of the $P$. trichocarpa genotype Nisqually-1 [21] and whole-transcriptome microarrays [22] enable the integration of genomic information with the quantitative genetic dissection of complex traits to uncover genes implicated in their variation using genetical genomics [23].

Here we report the genetic dissection of the variation in AR formation between two of the most economically important woody species in North America, $P$. deltoides and $P$. trichocarpa. We demonstrate the integration of traditional quantitative genetic methods with genomic information measured during the developmental program of AR formation, to identify major putative genes and hormonal biosynthesis pathways implicated in the control of this trait in the genus Populus.

\section{Results}

Adventitious rooting in the parental individuals

Adventitious root formation was characterized in a Populus pseudo-backcross population, referred hereafter as pedigree 52-124 [24, 25] (Fig. 1). The population was

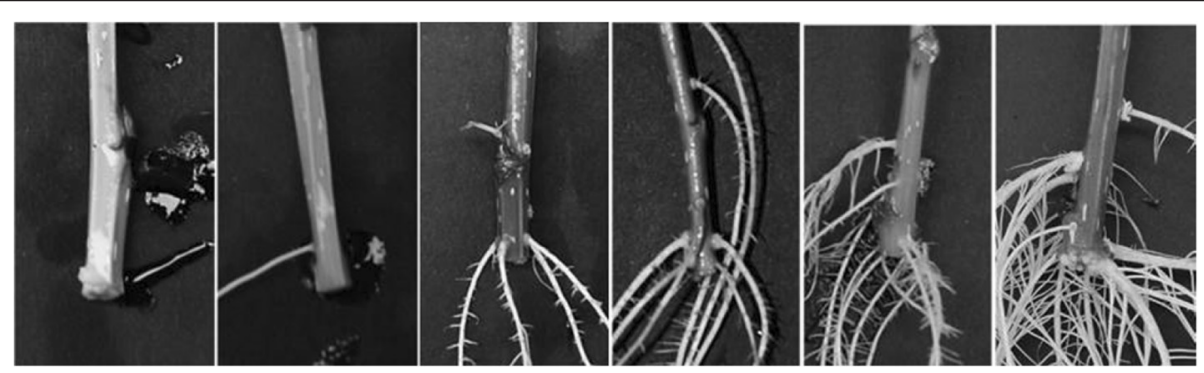

Fig. 1 Sample segregation of root number detected observed in family 52-124, after 18 days in hydroponic solution 
established by crossing a hybrid female parent (P. trichocarpa $\times P$. deltoides, genotype 52-225) with a pure $P$. deltoides (genotype D124). Cuttings from the parental individuals and 236 individuals from the progeny were placed in a hydroponic solution, and visible ARs were counted daily. Adventitious root primordia were observed after day 5 and over $85 \%$ of the cuttings had developed roots in the 18th day. In both parental individuals, AR formation started almost simultaneously, but the total number of visible roots was significantly lower $(P<0.01)$ in the pure $P$. deltoides male parent until the 17th day, in comparison to the hybrid female parent (Additional file 1). After the 17th day, the difference in the number of roots observed in the two parents was no longer significant. Therefore, both parents appear to have a similar capacity to develop AR, but there is a delayed development in $P$. deltoides relative to the hybrid parent ( $P$. trichocarpa $\times P$. deltoides).

\section{Quantitative genetic control of adventitious root formation} The broad-sense heritability $\left(\mathrm{H}^{2}\right)$ was calculated to estimate the extent of AR formation that is genetically controlled in pedigree 52-124, following previous studies that suggested high genetic control for the trait in Populus [26-28]. In this study, the heritability for number of roots was moderate $\left(H^{2}=0.27-0.34\right)$, similar to other complex traits previously analyzed in this pedigree [25].

In addition to root count, several architectural traits were measured in roots harvested after cuttings were maintained 18 days in hydroponic solution. The traits analyzed included total root length, surface area, volume and average diameter of total roots, and number of first order (primary) roots and root branches. All root architectural traits and total dry-weight showed transgressive segregation (Additional file 2). For these architectural traits, the heritability ranged from 0.12 for length of root branches to 0.26 for average diameter (Table 1 ).

Quantitative trait loci (QTL) analyses were performed for root architectural traits and for the number of roots counted after 18 days in hydroponic culture, using the genetic map of the hybrid mother [22]. For root architectural traits and root biomass, 15 QTLs were detected on the mother map, and the phenotypic variation explained by each QTL ranged from 6 to $11 \%$ (Additional file 3). Quantitative trait loci for number of ARs mapped consistently in the same intervals of linkage group (LG) II and LG XIV, and explained 7-10 \% of the phenotypic variation (Fig. 2 and Additional file 4). The logarithm of odds (LOD) score of these QTL reached 5.60 (QTL in LG II) and 4.99 (QTL in LG XIV). The QTL on LG II spans 34.89 centimorgans (cM) and includes 380 genes, while the QTL on LG XIV span $26.81 \mathrm{cM}$, with 241 genes. For identification of elements that regulate the number of ARs, further analysis focused on genes located within both QTL intervals on LG II and LG XIV.

\section{Transcriptome analysis of individuals with alternative alleles at the AR QTL}

To define putative regulators of AR formation, we searched for transcripts within QTLs on both LG II and XIV that were differentially regulated between individuals carrying the alternative parental alleles. This analysis assumes that genetic differences between individuals that inherited alternative QTL alleles results in differences in gene expression that impact $A R$ - i.e. the trait is at least partially controlled by differences in

Table 1 Broad sense pedigree heritability estimates for adventitious root-related phenotypes measured

\begin{tabular}{|c|c|c|c|c|}
\hline & Phenotypic values & Mean \pm SD & & \\
\hline Trait & P. trichocarp $a \times P$. deltoides & P. deltoides & Progeny Variation (Mean \pm SD) & Broad sense heritability $H^{2} \pm S D$ \\
\hline \multicolumn{5}{|l|}{ Root architectural traits } \\
\hline Total root length $(\mathrm{cm})$ & $56.5 \pm 6.2$ & $86.4 \pm 9.0$ & $123.1 \pm 94$ & $0.153 \pm 0.051$ \\
\hline Total root surface area $\left(\mathrm{cm}^{2}\right)$ & $10.0 \pm 1.1$ & $23.4 \pm 2.4$ & $25.1 \pm 19$ & $0.169 \pm 0.052$ \\
\hline Total root volume $\left(\mathrm{cm}^{3}\right)$ & $0.14 \pm 0.1$ & $0.51 \pm 0.1$ & $0.42 \pm 0.3$ & $0.185 \pm 0.053$ \\
\hline Average diameter (mm) & $0.56 \pm 0.2$ & $0.93 \pm 0.1$ & $0.70 \pm 0.2$ & $0.236 \pm 0.055$ \\
\hline Length of root branches $(\mathrm{cm})$ & $25.6 \pm 10.2$ & $28.5 \pm 8.6$ & $55.7 \pm 49$ & $0.115 \pm 0.049$ \\
\hline Surface area of root branches $\left(\mathrm{cm}^{2}\right)$ & $2.90 \pm 0.3$ & $2.40 \pm 0.1$ & $4.33 \pm 3.8$ & $0.117 \pm 0.049$ \\
\hline Volume of root branches $\left(\mathrm{cm}^{3}\right)$ & $0.28 \pm 0.8$ & $0.20 \pm 0.6$ & $0.034 \pm 0.03$ & $0.120 \pm 0.049$ \\
\hline Total length of primary roots (cm) & $30.8 \pm 9.1$ & $57.6 \pm 8.2$ & $67.1 \pm 50$ & $0.193 \pm 0.053$ \\
\hline Surface area of primary roots $\left(\mathrm{cm}^{2}\right)$ & $6.90 \pm 1.5$ & $20.1 \pm 2.5$ & $19.4 \pm 15$ & $0.187 \pm 0.053$ \\
\hline Volume of primary roots $\left(\mathrm{cm}^{3}\right)$ & $0.19 \pm 0.1$ & $0.61 \pm 0.1$ & $0.49 \pm 0.4$ & $0.177 \pm 0.053$ \\
\hline Number of adventitious roots (day 18) & $7.36 \pm 0.8$ & $4.92 \pm 0.5$ & $5.92 \pm 4.0$ & $0.341 \pm 0.056$ \\
\hline Root biomass (mg) & $4.73 \pm 0.9$ & $27.5 \pm 3.2$ & $19.3 \pm 20$ & $0.144 \pm 0.051$ \\
\hline
\end{tabular}




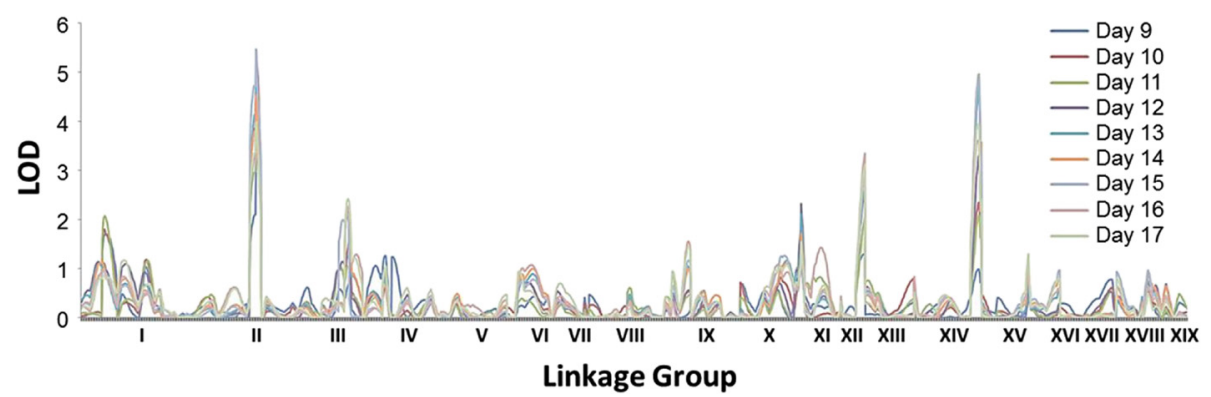

Fig. 2 Genome-wide composite interval mapping scan for number of roots detected in family 52-124, 9-17 days after cuttings were placed in hydroponic solution

transcript regulation. Gene expression in three individuals carrying alleles originated from the $P$. trichocarpa grandparent (UF352, UF498 and UF926, referred hereafter as the PtQTL genotype category) was contrasted with those with alleles from the $P$. deltoides grandparent (UF717, UF209 and UF912 or the PdQTL genotype category). These individuals were randomly selected among those that inherited the QTL flanking markers from either the $P$. trichocarpa grandparent (PtQTL genotype category) or the $P$. deltoides grandparent (PdQTL genotype category). For this transcriptome analysis, 25 cuttings of each of the six selected individuals were grown in hydroponic solution, and basal $(1 \mathrm{~cm})$ cutting sections from four biological replicates of each individual were collected at each of five time points $(0,24,48,96$ and $192 \mathrm{~h}$ after cuttings were harvested). We emphasized sample collection in the first $96 \mathrm{~h}$ because previous studies suggest that AR formation initiates within that period [29]. Five additional biological replicates of each individual were maintained in hydroponic growth conditions until day 12 , and confirmed that root development was consistent with the phenotype observed in the QTL detection experiment (Fig. 3). The transcriptome response of cuttings in hydroponic solution was assessed by whole-transcriptome

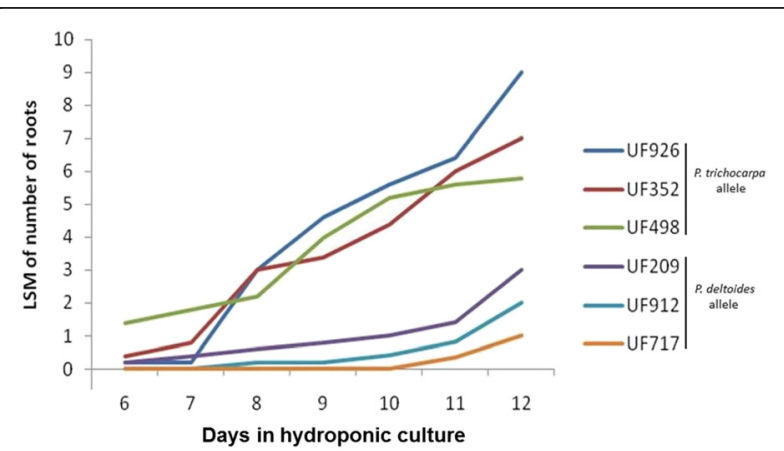

Fig. 3 Least square means number of adventitious roots developed on extreme genotypes selected for gene expression analysis microarrays developed previously [22, 24]. The microarray data generated for each gene was evaluated separately using analysis of variance (ANOVA) with time (0, 24, 48, 96 and 192 h), genotype (UF209, UF352, UF498, UF717, UF912 and UF926) and genotype $\times$ time interactions treated as fixed effects (see Methods). For each gene, an ANOVA F-test was carried out to identify if there were significant differences in expression among times of sample collection (0, 24, 48, 96 and 192 h). Significance was determined based on a false discovery rate (FDR) of $5 \%$. In addition, expression of each gene was compared between the individuals in the $P d \mathrm{QTL}$ genotype category (UF717, UF209 and UF912) and the individuals in the PtQTL genotype category (UF352, UF498 and UF926), at each time of sample collection. This analysis was carried out to assess the effect of the AR QTL on gene expression using a $5 \%$ FDR significance threshold, and is referred hereafter as the QTL genotype effect. We focused all further analysis on two comparisons: (1) time effect, and (2) QTL genotype effect.

\section{Time effect}

The F-test for the effect of the time of sample collection identified 26,121 putative genes as significantly differently expressed (FDR $<5 \%$ ) between at least two time points in the experiment (Additional file 5). To define the time at which the most significant changes in gene expression occurred, a comparison of gene expression between consecutive time points (i.e. time points $0-24$, 24-48, 48-96 and 96-192 h) was performed (Additional file 6). Most differences in transcript levels occurred during the first $24 \mathrm{~h}$ of the experiment (i.e. between 0 and $24 \mathrm{~h}$ ). Such extensive changes in the transcriptome are likely associated with stress and wounding responses that occurs immediately upon harvest of cuttings and their placement in the hydroponic solution, instead of solely due to the AR development. On the other hand, only ten genes were differentially regulated between 96 and $192 \mathrm{~h}$ in hydroponic culture, suggesting that these two time points are within the same rooting phase. 


\section{QTL genotype effect}

Next we sought to identify genes differentially expressed between individuals in the PdQTL and PtQTL categories, at each time point. The aim of this comparison is to identify genes that are differentially regulated during the initial development of AR, between individuals that carry alternative alleles at the QTL that control the trait. Genes differentially regulated between individuals in the $P d \mathrm{QTL}$ and PtQTL categories, at any of the time points, and located within the QTL intervals, represent candidate AR regulators. We identified 1929 genes differentially regulated between genotypes in the PtQTL and $P d \mathrm{QTL}$ categories, in at least one of the time points of tissue collection (Additional file 7). Of these 1929 genes, 81 are located within the QTL intervals on LG II and XIV. Among these, two putative homologues of genes that encode for enzymes of the tryptophan biosynthesis pathway are of particular interest: POPTR_0002s04640 and POPTR_0002s02770. Tryptophan is a precursor of auxin $[30,31]$, the primary hormone regulator of AR formation. The gene POPTR_0002s04640 is a putative homolog of TSA1 (TRYPTOPHAN SYNTHASE ALPHA CHAIN). The enzyme encoded by TSA1 catalyzes the conversion of indole-3-glycerolphosphate to indole, the second to last reaction in the tryptophan biosynthesis, and showed lower expression among the better rooting individuals of the PtQTL category at 48 and $192 \mathrm{~h}$. POPTR_0002s02770, the putative homolog of the Arabidopsis gene SUR2 (SUPERROOT2), was significantly more highly expressed among individuals in the PdQTL category, compared to those in PtQTL, at 192 h. A SUR2 knockout mutant has been shown to cause auxin overproduction and an abnormally high number of adventitious roots [32, 33]. In the analysis of the time effect, POPTR_0002s02770 was also significantly differentially expressed between times 0 and $24 \mathrm{~h}$, among individuals in both the PdQTL and PtQTL categories.

Other genes implicated in phytohormone response and located within the target QTL intervals were also differentially regulated between individuals in the $P d \mathrm{QTL}$ and $P t Q T L$ categories, at several time points. For instance, at 96 and $192 \mathrm{~h}$, higher expression was observed for the gene POPTR_0002s02420, which is a homolog of Arabidopsis GA-STIMULATED TRANSCRIPT 1 (GASA1) among individuals of the PdQTL category. GASA1 is involved in response to gibberellins stimulus, brassinosteroid, abscisic acid stimulus and unidimensional cell growth [34]. At 48 and 96 h, a homolog of the Arabidopsis gene ETO1LIKE PROTEIN 1 (EOL1), POPTR_0002s04910, was more highly expressed in individuals of the PtQTL category. EOL1 encodes a paralog of ETHYLENE-OVERPRODU$C E R 1$, which is a negative regulator of the gene 1-AMINOCYCLOPROPANE-1-CARBOXYLATE SYNTHASE 5, a key enzyme in ethylene biosynthesis pathway [35].
Clustering the difference in transcriptome response of PtQTL and PdQTL genotypes

Genes differentially regulated between individuals in the PtQTL and PdQTL categories are likely to be part of an orchestrated response that distinguishes the two species in their ability to form AR. To uncover the differential functional responses, we clustered the 1929 genes differentially regulated between the PtQTL and PdQTL categories, based on the difference in transcript abundance between the two at each time point. Genes with a common pattern of differential regulation throughout the experiment were clustered using a Modulated Modularity Clustering (MMC) graph-based method [36]. Sixty clusters were identified, varying from 2 to 148 transcripts in size, and eight genes remained unclustered (Additional file 8 and Additional file 9).

\section{Transcription factor binding site analysis}

Of all the genes identified as differentially regulated between individuals in the PdQTL and PtQTL categories, and located within the QTL intervals, only POPTR_0002s02770 is a homolog to an Arabidopsis gene previously shown to control AR development (SUPERROOT2, Boerjan, 1995 [32]; Delarue et al., 1998 [33]). Because of the potential role of POPTR_0002s02770 in AR formation of poplars, we used the PLACE (plant cis-acting regulatory DNA elements) database to identify conserved motifs over represented in the cluster containing this gene. This analysis is constrained by the fact that it is solely based on motifs detected in P. trichocarpa, because a suitable reference genome sequence is not available for $P$. deltoides. A total of 48 genes grouped in the superroot2 cluster, and half of them were highly expressed in individuals of the PdQTL category while the other half were highly expressed in individuals of the PtQTL category. We hypothesized that genes in this cluster would share motifs related to hormone regulation, particularly auxin. A Fisher's exact test was performed to identify motifs in higher frequency in one of the two QTL categories, which identified 25 significant motifs (Table 2). Interestingly, all motifs were significantly $(P$-value $>0.05)$ enriched in the PtQTL category group of genes. Half of the motifs detected have been previously described to be associated with phytohormonal response, mainly auxin (6 motifs categories) but also ethylene, abscisic acid and gibberellins. Also, several motifs are directly related to rooting and wounding response. These results add evidence to the influence of these co-expressed genes in regulating adventitious root formation.

\section{Discussion and conclusions}

The capacity of plants to develop AR is extensively used by many industries and research segments to 
Table 2 Putative regulatory motifs in significantly higher frequency among genes belonging to the superroot2 cluster, that are more highly expressed in individuals that inherited the P. trichocarpa QTL allele (PtQTL category), based on Fisher's exact test

\begin{tabular}{|c|c|c|}
\hline Motif Sequence & $p$-value two side test & Annotation \\
\hline TGACY & 0.02854664 & ABA responsive element, $A B R E 3$ \\
\hline TTATT & 0.02854664 & highly active synthetic auxin response \\
\hline RTTTTR & 0.01348537 & highly active synthetic auxin response \\
\hline AGATC & 0.03372874 & ERE (ethylene responsive element) \\
\hline TTGAC & 0.03372874 & highly active synthetic auxin response \\
\hline CCTTT & 0.01817917 & highly active synthetic auxin respone \\
\hline AAAGAT & 0.00121528 & ERE (ethylene responsive element) \\
\hline TATTCT & 0.01943102 & highly active synthetic auxin response \\
\hline CAANNNNATC & 0.02472731 & highly active synthetic auxin response \\
\hline CAACA & 0.02854664 & ABA responsive element, ABRE3 \\
\hline GATAA & 0.02854664 & GARE (gibberellic acid responsive element) \\
\hline RGATY & 0.02854664 & MeJa-responsive element (MeJaRE) \\
\hline AATAAT & 0.03372874 & TATA Box \\
\hline TATTAAT & 0.00474209 & TATA Box \\
\hline AATAAA & 0.02854664 & TATA Box \\
\hline ACGTG & 0.04506551 & ACGT motif" related to root expression \\
\hline ATATT & 0.02854664 & RSE (root-specific element) \\
\hline CANNTG & 0.02854664 & ACGT motif related to root expression \\
\hline CAAACAC & 0.00985829 & RSR (root specific region) S000243 \\
\hline AATTAAA & 0.01817917 & WAR (wounding activating region) \\
\hline CWWWWWWWWG & 0.04047776 & Wound-responsive element (WRE) \\
\hline ATAGAA & 0.01943102 & WAR (wounding activating region) \\
\hline TGHAAARK & 0.0418575 & Binding site of wound-inducible \\
\hline ATATTTAWW & 0.01082454 & Wound-responsive element (WRE) \\
\hline GGTTAA & 0.04506551 & Stress responsive element (SRE) \\
\hline ATGGTA & 0.03390743 & Stress responsive element (SRE) \\
\hline GATAAG & 0.04970707 & Stress responsive element (SRE) \\
\hline TATTAG & 0.0418575 & SE2 (stem element 2) \\
\hline
\end{tabular}

propagate elite individuals selected in breeding programs or in natural populations. Significant economic losses are associated with cuttings producing poor quality root systems or complete failing to form them [1]. In this study we combined the genetic (QTL) analysis of a segregating population, with genome and transcriptome data to identify putative regulators of AR development in Populus. The analysis focused on the segregation of alleles from a hybrid of $P$. trichocarpa and $P$. deltoides in a mapping population. Previous observations identified the two species as being contrastingly distinct with respect to AR formation.

We detected a moderate heritability for most AR developmental traits analyzed, in line with similar studies in other Populus species [37, 38]. Only two QTL studies on AR development in Populus had been previously reported [26, 39]. Han and colleagues studied the quantitative genetic aspect of in vitro adventitious root formation and shoot regeneration, and Zhang et al. [26] used functional mapping to detect QTLs for number of roots and maximum root length measured at different time points. However, no common QTL were detected between those studies and the results reported here. Differences might be attributed to the use of families with distinct genetic backgrounds, growth conditions and type of cuttings.

The transcriptome data indicates that the largest number of genes is differentially regulated in the first $24 \mathrm{~h}$ after cuttings were harvested, regardless of the QTL allele inherited. This result is expected to be due to hormonal and gene regulation changes related to wound response and de-differentiation of the cells to a meristematic state capable of cell division. Expectedly, numerous genes up-regulated during the first $24 \mathrm{~h}$ reflect 
these changes. These includes CPC902 (CONDENSIN COMPLEX COMPONENTS SUBUNIT C), a homolog of the Arabidopsis gene SMC1 (STRUCTURAL MAINTENANCE OF CHROMOSOMES 1). SMC1 encodes for one of the proteins of the cohesion complex family [40], necessary for correct chromosome segregation during nuclear divisions, possibly indicating the initiation of cell divisions necessary for root meristem organization.

The identification of transcripts differentially regulated at different time points following the collecting of cuttings provides a broad, transcriptome overview of genes and pathways that may participate in wounding and cell de-differentiation, root induction and elongation [1]. However, it does not identify a defined set of genes or specific polymorphisms that are responsible for the phenotypic differences between AR formation in P. deltoides and $P$. trichocarpa. To achieve this goal we contrasted gene expression between individuals carrying alternative alleles that control AR formation, detected based on a QTL analysis, at each time point. Genes differentially regulated between individuals that inherited the alternative $P$. deltoides or $P$. trichocarpa QTL alleles were then evaluated for their position in the Populus genome, to detect those located within the QTL intervals. Among those genes, SUR2 undergoes a highly significant reduction in expression between the time the cuttings were taken and the first $24 \mathrm{~h}$ in hydroponic culture. This reduction in expression is independent on the genotype at the QTLs-it is observed in those individuals in the PtQTL and PdQTL categories. However, in the following time points (48-192 h), the levels of SUR2 remain low in the individuals that form AR early (PtQTL category) but rise steeply towards levels detected at $0 \mathrm{~h}$ in the poor AR developing individuals (PdQTL category) (Fig. 4a). Interestingly, poplar's putative homologue of TSA1, which encodes for the enzyme that catalyzes the conversion of indole-3-glycerolphosphate to indole, the second to last reaction in the tryptophan biosynthesis, is also located in the LG II QTL interval. TSA1 also shows lower expression among the better rooting individuals of the PtQTL category in later time points of 48 and $192 \mathrm{~h}$ (Fig. 4b). Taken together, the data suggests that poplar genotypes that are limited in AR formation could synthesize auxin indole-3-acetic acid (IAA) primarily through the tryptophan (Trp) pathway. However, much of the pathway flux appears directed towards synthesis of indole glucosinolates (IG) because on the overexpression of SUR2. On the contrary, genotypes that are efficient in AR formation down-regulate the synthesis of Trp (by down-regulating TSA1) and/or the diversion of the pathway towards synthesis of IG. The auxin IAA has long been postulated to be synthesized through multiple pathways [41], including a Trp-independent pathway [42]. Recently, an Arabidopsis indole synthase mutant defective in the Trp-independent auxin biosynthetic pathway was uncovered [43]. Gene expression in the poplar putative homologue was investigated in this study, but showed no significant difference in transcript levels among genoytpes, and over time.

Clearly, gene expression may not reflect protein level changes or other process (such as protein modifications) that could impact IAA biosynthesis. Other genes differentially regulated between individuals in the PtQTL and PdQTL categories, and located within the AR QTL interval, may also be relevant and should be considered upon further analysis. Furthermore, although significant differences in gene expression were detected in SUR2 and other genes related to IAA biosynthesis, the biological impact of these changes can only be assessed by further experimentation that is beyond the scope of the research described here. Small differences in gene expression may be significant statistically, but have no or limited biological impact. Despite these concerns, this study raises an attractive hypothesis that the difference between AR formation in $P$. deltoides and $P$. trichocarpa is driven by difference in the expression of genes in the IAA biosynthesis pathway, possibly under the control of the poplar homologues of SUR2 and TSA1.
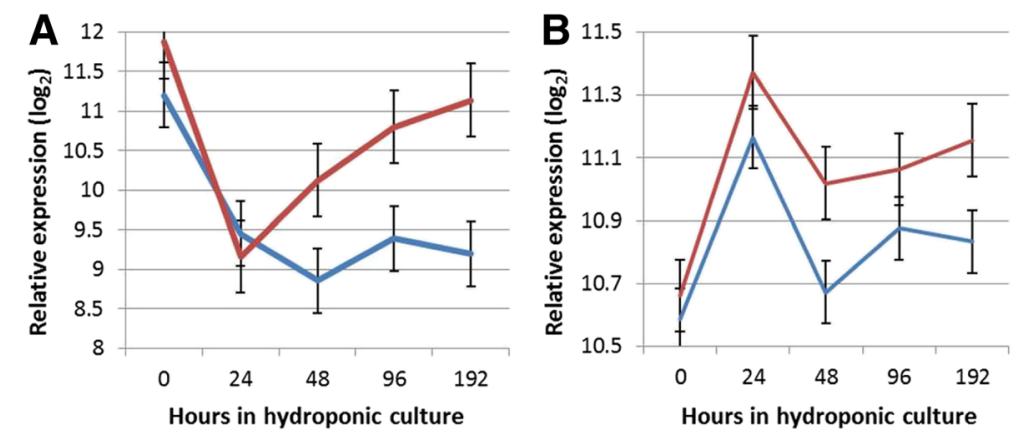

Fig. 4 Relative expression level (log2) of SUR2 (Panel a) and TSA1 (Panel b) at different time points, measured as the least square mean of individuals in the PtQTL category (blue line) and the PdQTL category (red line). Error bars show the standard error 


\section{Methods}

\section{Plant material and phenotypic measurements}

The pedigree (52-124) used in this study is a pseudobackcross between the hybrid female parent 52-225 $[P$. trichocarpa (clone 93-968) $\times P$. deltoides (clone ILL-101)] and the unrelated male parent D124 ( $P$. deltoides), established by the Natural Resources Research Institute of the University of Minnesota. The parent D124 is from northern Minnesota. The P. trichocarpa parent of the hybrid came from western Washington, whereas the $P$. deltoides parent material originated in Illinois. Twelve centimeters $(\mathrm{cm})$ apical cuttings were collected from 234 individuals of pedigree 52-124, as well as the parental individuals. Cuttings were placed in $58 \times 41 \times 15 \mathrm{~cm}$ containers, with up to 59 cuttings per container, and maintained in hydroponic culture $\left(\mathrm{H}_{2} \mathrm{O}\right.$ buffered at $\mathrm{pH}$ 5.7, with $0.5 \mathrm{~g} \mathrm{~L}^{-1}$ of MES) for the duration of the experiments. The experimental design was an incomplete block design with four blocks and three replications, for a total of 708 cuttings. Root emergence was recorded daily at the same time $(10 \mathrm{am})$, until the 18th day of culture. After 18 days in hydroponic solution, roots were harvested, scanned using Scanner CanoScan LiDE 600 F (Cannon) and dried for measurement of total dry-weight. The scanned roots were analyzed using WinRHIZO Pro (Regent Instruments Inc.) for total root length, surface area, volume and average diameter of total roots, first order (primary) roots and root branches. Because the experiment was conducted in a closed environment and all material was destroyed upon completion of measurements, permits are not required by the existing legislation. A public collection established at the University of Florida provides permanent access to the material utilized in this study.

\section{Statistical analysis}

Covariance parameters were estimated for all traits using PROC MIXED (SAS Institute Inc. 9.2 ${ }^{\circ}$ 2004, Cary, NC, USA), considering all variables random in the following model:

$$
\gamma_{i j k l}=\mu+\alpha_{i}+\beta_{j}+\gamma_{k(j)}+e_{i j k l}
$$

where $\gamma_{i j k l}$ is the phenotypic value of the $i$ th genotype in the $j$ th block within the $k$ th replication, $\mu$ is the overall mean, $\alpha_{i}$ is the random effect of the genotype; $\beta_{j}$ is the random effect of replication, $\gamma_{k(j)}$ is the random effect of incomplete block (within replication) and $e_{i j k l}$ is the residual error.

Broad-sense pedigree heritability was calculated using the covariance parameter estimates in the following formula:

$$
H^{2}=\frac{\sigma_{c}^{2}}{\sigma_{c}^{2}+\sigma_{e}^{2}}
$$

where $\sigma_{c}^{2}$ and $\sigma_{e}^{2}$ are the variance components corresponding to genotype and residual effect across the three replications, respectively.

A log transformation was applied to all traits, except for number of roots. Least-square means used in the QTL analysis were calculated by including clone as a fixed effect in the model, using PROC MIXED.

\section{QTL analysis}

QTLs for root-related traits were identified based on a linkage map previously described [22, 25]. The linkage map consists of 181 markers chosen on the basis of homogenous distribution in the hybrid female parent. The map had an average density of one marker every 16 cM. QTLs were identified using composite interval mapping [44] in Windows QTL Cartographer v.2.5 using standard model 6 with walk speed of $2 \mathrm{cM}$. A genomewide significance level of $P<0.05$ was established based on 1000 permutations [45].

\section{Selection of individuals with alternative alleles in QTL regions}

Quantitative trait loci for the number of roots were consistently mapped on LG II and XIV (see Results). We classified each individual depending on the allele (P. trichocarpa or $P$. deltoides) that was observed in both QTL regions. Four categories were defined: (1) individuals carrying $P$. deltoides or (2) P. trichocarpa alleles at both QTLs, and (3) individuals carrying $P$. trichocarpa alleles at the QTL in LGII and P. deltoides alleles in QTL on LG XIV, and (4) vice-versa. Individuals with recombination between markers flanking in each of the two QTL were not grouped into any of the categories. As expected, individuals carrying $P$. trichocarpa alleles in both QTL regions (PtQTL category) generally had more roots than those carrying the $P$. deltoides alleles in those intervals (PdQTL category). For these six individuals we collected $12 \mathrm{~cm}$-long cuttings and established them in the same hydroponic conditions used previously in the QTL detection experiment. The number of new roots formed in these individuals was recorded daily for 12 days, and samples were collected for transcriptome analysis.

\section{Tissue sampling for microarray analysis}

To measure gene expression during adventitious root formation in the three selected individuals from each QTL category (PtQTL and PdQTL), a $1 \mathrm{~cm}$ section, measured from the base of each cutting, was collected at 0, 24, 48, 96 and $192 \mathrm{~h}$ after placing them in the hydroponic solution. Samples were flash-frozen in liquid nitrogen for posterior RNA extraction. Four biological 
replicates were collected from each individual, at each time point. In addition, five biological replicates of each individual were maintained in hydroponic growth conditions until day 12 to verify that the root development was consistent with the phenotype observed in the QTL detection experiment.

\section{RNA extraction, CDNA synthesis and labeling}

Total RNA was extracted [46] from the bottom $1 \mathrm{~cm}$ stem section collected from each sample. The sample included xylem, phloem and bark. RNA was purified using RNeasy Mini Kit columns (Qiagen), and DNase treated with RNase-Free DNase set (Qiagen). RNA quality was evaluated in $1 \% \mathrm{w} / \mathrm{v}$ agarose gels. RNA was amplified and cRNA synthesized and labeled using Two Dyes Agilent Low Input Quick Amp Labeling Kit (Agilent). The microarray platform used consisted of single 60-mer probes designed for each of 43,803 annotated gene models from the sequenced genome of $P$. trichocarpa (National Center for Biotechnology Information Gene Expression Omnibus Platform GPL20736). These probes were previously selected for being suitable for analysis of gene expression in this mapping population [22].

\section{Microarray experimental design and data analysis}

A total of 60 microarrays were used in the transcriptome analysis. Gene expression of each of six individuals was analyzed in five time points $(0,24,48,96$ and $192 \mathrm{~h})$, with four biological replicates per individual and time point. The design was selected to favor contrasting gene expression of samples from different QTL categories (PtQTL and PdQTL) at each time point, as well as samples from the same individual collected from different time points. Data is stored in the National Center for Biotechnology Information Gene Expression Omnibus Series GSE71630. Median values of signal intensities were quantile normalized [47] and $\log _{2}$ transformed. Normalized signals were analyzed in SAS 9.2 (SAS Institute Inc. $9.2^{\circ} 2004$, Cary, NC, USA) using a mixedmodel ANOVA with genotype and genotype $\times$ time interactions as fixed effects, and microarray as random effect. Differences in expression between the group of individuals from the PtQTL and PdQTL categories were estimated at each time point, and the significance was determined based on a false discovery rate (FDR) of $5 \%$ [48]. Genes showing a similar pattern of expression differences between individuals from the PtQTL and PdQTL categories, at all time points, were clustered using a Modulated Modularity Clustering graph-based technique using Spearman correlation [36].

\section{Annotation}

Populus gene model transcript sequences were annotated by searching for sequence similarities using BLASTx against Populus (JGI v.1.1 and v2.2) and The Arabidopsis Information Resource (TAIR v8.0) gene models.

\section{Transcription factor binding sites analysis}

Promoter sequences upstream of the start codon of $P$. trichocarpa gene models were previously extracted [24] to identify presence and absence of common plant cis-acting elements. The cluster containing gene POPTR_0002s02770, Arabidopsis homolog of SUR2 was divided into two groups based on the gene expression pattern i.e., genes being more highly expressed in the PtQTL or PdQTL category. PLACE (plant cis-acting regulatory DNA elements) database of nucleotide motifs [49] was used to identify conserved motifs being over represented in each of these groups and also infer functional roles in coregulated genes. Two-sided fisher exact test was performed in SAS (SAS Institute Inc. 9.2 2004, Cary, NC, USA) using PROC FREQ to test over-representation of a specific motif in genes being highly expressed among individuals of the $P d \mathrm{QTL}$ category against genes highly expressed among individuals of the PtQTL category within SUR2 cluster.

\section{Availability of supporting data}

The microarray data is publically available in the National Center for Biotechnology Information Gene Expression Omnibus under the accession numbers GSE71630.

\section{Additional files}

\begin{abstract}
Additional file 1: Cumulative number of roots formed in the parents of pedigree 52-124. Least-square means of number of adventitious roots developed on the female hybrid parent Populus trichocarpa $\times P$. deltoides 52-225 (red line), and the unrelated male parent $P$. deltoides D124 (blue line), maintained in hydroponic solution for 25 days. (DOCX 67 kb)
\end{abstract}

Additional file 2: Frequency distribution of root architectural and biomass traits. Frequency distribution of least-square means of root architectural and biomass traits measured on 225 individuals of pedigree 52-124. Parents 'P. deltoides' (D) and (P. trichocarpa $\times P$.deltoides $) \times P$.deltoides (TD) are indicated. Measurements were made after 18 days of growth in hydroponic solution. Traits are total root length $(\mathrm{cm}$, panel A), tota root surface area $\left(\mathrm{cm}^{2}\right.$, panel $\left.\mathrm{B}\right)$, total root volume $\left(\mathrm{cm}^{3}\right.$, panel $\left.\mathrm{C}\right)$, average diameter ( $\mathrm{mm}$, panel D), length of root branches $(\mathrm{cm}$, panel E), surface area of root branches $\left(\mathrm{cm}^{2}\right.$, panel F), volume of root branches $\left(\mathrm{cm}^{3}\right.$, panel $\left.\mathrm{G}\right)$, total length of primary roots $(\mathrm{cm}$, panel $\mathrm{H})$, surface area of primary roots $\left(\mathrm{cm}^{2}\right.$, pane I), volume of primary roots $\left(\mathrm{cm}^{3}\right.$, panel J), number of adventitious roots at 18 days (panel $\mathrm{K}$ ) and root biomass (mg, panel L). (DOCX $326 \mathrm{~kb}$ )

Additional file 3: QTL detected for root architecture traits and root biomass. Phenotypic variance explained by each QTL interval detected for root architecture traits and root biomass. Respective linkage group $(\mathrm{LG})$, flanking marker location, LOD score and origin of positive allele. (DOCX $17 \mathrm{~kb}$ )

Additional file 4: QTL detected for the trait number of adventitious roots. Phenotypic variance explained by each QTL interval identified for number of root traits. Respective linkage group (LG), flanking markers location, LOD peak and origin of positive allele. (DOCX $16 \mathrm{~kb}$ )

Additional file 5: Genes differentially regulated among time point of sample collection. Genes differentially expressed among times of sample 
collection. The table describes the name of each poplar gene based on all three main annotations of the poplar genome (v. 1.1, v. 2.2 and v. 3.0), the name of the probe on the microarray, the FDR adjusted p-value for the F-test of the effect of TIME in the ANOVA, and the estimate of the effect of TIME at 0, 24, 48, 96 and 192 h. (XLSX $3466 \mathrm{~kb})$

Additional file 6: Differentially regulated genes between time points. Number of genes differentially expressed when contrasting consecutive time points. (DOCX $44 \mathrm{~kb}$ )

Additional file 7: Differentially regulated genes between PdQTL and PtQTL categories, at each time point. The table describes the name of each poplar gene based on all three main annotations of the genome (v. 1.1, v. 2.2 and v. 3.0), the name of the probe on the microarray, and the gene expression difference $\left(\log _{2}\right)$ between individual in the $P d Q T L$ and PtQTL categories. Data is presented only for those genes and time points were the difference in expression between $P d Q T L$ and $P t Q T L$ was significant for an FDR adjusted p-value of $5 \%$. (XLSX $139 \mathrm{~kb}$ )

Additional file 8: Clustering the difference in transcriptome response of PtQTL and PdQTL genotypes. Modulated Modularity Clustering of genes displaying a similar pattern of expression differences between genotypes from the PtQTL and PdQTL categories, at all time points. (DOCX $25 \mathrm{~kb}$ )

Additional file 9: Gene membership of Modulated Modularity Clusters. Genes membership of Modulated Modularity Clusters detected based on genes displaying a similar pattern of expression differences between genotypes from the PtQTL and PdQTL categories, at all time points. (XLSX $262 \mathrm{~kb})$

\section{Abbreviations}

AR: adventitious roots; CIM: composite interval mapping; cM: centimorgan; FDR: false-discovery rate; IAA: indole-3-acetic acid; LG: linkage group; LOD: logarithm of the odds; MMC: modulated modularity clustering; QTL: quantitative trait locus; SUR2: SUPERROOT2; Trp: tryptophan; TSA1: TRYPTOPHAN SYNTHASE ALPHA CHAIN.

\section{Competing interests}

The author's declare no competing interests.

\section{Authors' contribution}

CR participated in the analysis and drafted the manuscript. CS carried out the data collection and performed the statistical analysis. DD participated on the phenotypic data analysis and construction of the genetic map. EV participated on the phenotypic data analysis and construction of the genetic map. CRDBN participated in the microarray experiment. CD participated in the design of the study and collection of the data. MK conceived the study, participated in its design and helped to draft the manuscript. All authors read and approved the final manuscript.

\section{Acknowledgements}

This work was supported by the Department of Energy, Office of Science, Office of Biological and Environmental Research, grant awards numbers DE-FG02-05ER64114 and DE-SC0003893 (to MK). We acknowledge all the staff and students from the Forest Genomics Lab at the University of Forida for the help with the data collection and Dr. Dudley Huber for the help with the experimental design. We also acknowledge the two anonymous reviewers, who provided valuable suggestions that improved the manuscript.

\footnotetext{
Author details

${ }^{1}$ School of Forest Resources and Conservation, University of Florida, P.O. Box 110410, Gainesville, FL 32611, USA. ²Plant Molecular and Cellular Biology Graduate Program, University of Florida, P.O. Box 110690, Gainesville, FL 32611, USA. ${ }^{3}$ University of Florida Genetics Institute, University of Florida, P.O. Box 103610, Gainesville, FL 32611, USA. ${ }^{4}$ Present Address: Monsanto Company, 700 Chesterfield Pkwy W, Chesterfield, MO 63017, USA. ${ }^{5}$ Present Address: Seminis, Inc., 37437 State Highway 16, Woodland, CA 95695, USA. ${ }^{6}$ Present Address: Universidade Federal de Goiás, Av. Esperança s/n' Goiânia, GO 74001-970, Brazil.
}

Received: 12 August 2015 Accepted: 6 March 2016 Published online: 16 March 2016

\section{References}

1. De Klerk G-J, van der Krieken W, de Jong JC. Review the formation of adventitious roots: New concepts, new possibilities. Vitr Cell Dev Biol Plant. 1999:35:189-99.

2. Srivastava LM. Plant growth and development: hormones and environment. Oxford: Academic Press; 2002.

3. Lovell P, White J. Anatomical changes during adventitious root formation. In: New root formation in plants and cuttings. 1985. p. 111-40.

4. Klerk G-J. Rooting of microcuttings: Theory and practice. Vitr Cell Dev Biol Plant. 2002;38:415-22.

5. Ahkami AH, Lischewski S, Haensch K-T, Porfirova S, Hofmann J, Rolletschek H, Melzer M, Franken P, Hause B, Druege U, Hajirezaei MR. Molecular physiology of adventitious root formation in Petunia hybrida cuttings: involvement of wound response and primary metabolism. New Phytol. 2009;181:613-25.

6. Da Costa CT, de Almeida MR, Ruedell CM, Schwambach J, Maraschin FS, Fett-Neto AG. When stress and development go hand in hand: main hormonal controls of adventitious rooting in cuttings. Front Plant Sci. 2013;4:133.

7. Della Rovere F, Fattorini L, D'Angeli S, Veloccia A, Falasca G, Altamura MM. Auxin and cytokinin control formation of the quiescent centre in the adventitious root apex of Arabidopsis. Ann Bot. 2013;112:1395-407.

8. Sun W-Q, Bassuk NL. Auxin-induced ethylene synthesis during rooting and inhibition of budbreak of 'Royalty' rose cuttings. J Amer Soc Hort Sci. 1993; 118:638-43

9. Ramírez-Carvajal GA, Morse AM, Dervinis C, Davis JM. The cytokinin type-B response regulator PtRR13 is a negative regulator of adventitious root development in Populus. Plant Physiol. 2009;150:759-71.

10. Mauriat M, Petterle A, Bellini C, Moritz T. Gibberellins inhibit adventitious rooting in hybrid aspen and Arabidopsis by affecting auxin transport. Plant J. 2014:78:372-84.

11. Niu S, Li Z, Yuan H, Fang P, Chen X, Li W. Proper gibberellin localization in vascular tissue is required to regulate adventitious root development in tobacco. J Exp Bot. 2013;64:3411-24.

12. Sun $H$, Tao J, Hou M, Huang $S$, Chen $S$, Liang $Z$, Xie T, Wei Y, Xie X, Yoneyama $\mathrm{K}, \mathrm{Xu} \mathrm{G}$, Zhang Y. A strigolactone signal is required for adventitious root formation in rice. Ann Bot. 2015:115:1155-62

13. Rasmussen A, Beveridge CA, Geelen D. Inhibition of strigolactones promotes adventitious root formation. Plant Signal Behav. 2012:7:694-7.

14. Borralho NMG, Wilson PJ. Inheritance of initial survival and rooting ability in Eucalyptus globulus Labill. stem cuttings. Silvae Genet. 1994:43:238-42.

15. Grattapaglia D, Bertolucci FL, Sederoff RR. Genetic mapping of QTLs controlling vegetative propagation in Eucalyptus grandis and E. urophylla using a pseudo-testcross strategy and RAPD markers. Theor Appl Genet. 1995:90:933-47.

16. Dickmann D, Hendrick R. Modeling adventitious root system development in trees: clonal poplars. In: Davis T, Haissig B, editors. Biology of adventitious root formation. New York: Plenum Press; 1994. p. 203-18.

17. Bradshaw HD, Ceulemans R, Davis J, Stettler R. Emerging model systems in plant biology: Poplar (Populus) as a model forest tree. J Plant Growth Regul. 2000;19:306-13.

18. Taylor G. Populus: arabidopsis for forestry. Do we need a model tree? Ann Bot. 2002;90:681-9.

19. Wullschleger SD, Jansson S, Taylor G. Genomics and forest biology: Populus emerges as the perennial favorite. Plant Cell. 2002;14:2651-5.

20. Brunner AM, Busov VB, Strauss SH. Poplar genome sequence: functional genomics in an ecologically dominant plant species. Trends Plant Sci. 2004; 9:49-56.

21. Tuskan GA, Difazio S, Jansson S, Bohlmann J, Grigoriev I, Hellsten U, et al. The genome of black cottonwood, Populus trichocarpa (Torr. \& Gray). Science. 2006:313:1596-604.

22. Drost DR, Novaes E, Boaventura-Novaes C, Benedict Cl, Brown RS, Yin T, Tuskan GA, Kirst M. A microarray-based genotyping and genetic mapping approach for highly heterozygous outcrossing species enables localization of a large fraction of the unassembled Populus trichocarpa genome sequence. Plant J. 2009;58:1054-67.

23. Jansen RC, Nap JP. Genetical genomics: the added value from segregation. Trends Genet. 2001;17:388-91.

24. Drost DR, Benedict Cl, Berg A, Novaes E, Novaes CRDB, Yu Q, Dervinis C, Maia JM, Yap J, Miles B, Kirst M. Diversification in the genetic architecture of gene expression and transcriptional networks in organ differentiation of Populus. Proc Natl Acad Sci U S A. 2010;107:8492-7. 
25. Novaes E, Osorio L, Drost DR, Miles BL, Boaventura-Novaes CRD, Benedict C, Dervinis C, Yu Q, Sykes R, Davis M, Martin TA, Peter GF, Kirst M. Quantitative genetic analysis of biomass and wood chemistry of Populus under different nitrogen levels. New Phytol. 2009;182:878-90.

26. Zhang $B$, Tong $C$, Yin $T$, Zhang $X$, Zhuge $Q$, Huang $M$, Wang $M$, Wu R. Detection of quantitative trait loci influencing growth trajectories of adventitious roots in Populus using functional mapping. Tree Genet Genomes. 2009;5:539-52.

27. Wilcox JR, Farmer RE. Heritability and C effects in early root growth of eastern cottonwood cuttings. Heredity (Edinb). 1968;23:239-45.

28. Ying CC, Bagley WT. Variation in rooting capability of Populus deltoides. Silvae Genet. 1977;26:204-7.

29. De Klerk G-J, Keppel M, Brugge JT, Meekes H. Timing of the phases in adventitous root formation in apple microcuttings. J Exp Bot. 1995;46:965-72.

30. Mashiguchi K, Tanaka K, Sakai T, Sugawara S, Kawaide H, Natsume M, Hanada A, Yaeno T, Shirasu K, Yao H, McSteen P, Zhao Y, Hayashi K, Kamiya $\mathrm{Y}$, Kasahara H. The main auxin biosynthesis pathway in Arabidopsis. Proc Natl Acad Sci U S A. 2011;108:18512-7.

31. Mano Y, Nemoto K. The pathway of auxin biosynthesis in plants. J Exp Bot. 2012;63:2853-72.

32. Boerjan W. superroot, a recessive mutation in Arabidopsis, confers auxin overproduction. Plant Cell Online. 1995;7:1405-19.

33. Delarue M, Prinsen E, Onckelen HV, Caboche M, Bellini C. Sur2 mutations of Arabidopsis thaliana define a new locus involved in the control of auxin homeostasis. Plant J. 1998;14:603-11.

34. Bouquin T, Meier C, Foster R, Nielsen ME, Mundy J. Control of specific gene expression by gibberellin and brassinosteroid. Plant Physiol. 2001;127:450-8.

35. Christians MJ, Gingerich DJ, Hansen M, Binder BM, Kieber JJ, Vierstra RD. The BTB ubiquitin ligases ETO1, EOL1 and EOL2 act collectively to regulate ethylene biosynthesis in Arabidopsis by controlling type-2 ACC synthase levels. Plant J. 2009:57:332-45.

36. Stone EA, Ayroles JF. Modulated modularity clustering as an exploratory tool for functional genomic inference. PLoS Genet. 2009;5:e1000479.

37. Riemenschneider DE, Bauer EO. Quantitative genetic analysis of adventitious root forming ability in populus trichocarpa (Torr Et. Gray). In: Altman A, Waisel Y, editors. Biology of Root Formation and Development SE - 36. Volume 65. New York, US: Springer; 1997. p. 193-201. [Basic Life Sciences].

38. Zalesny RSJ, Riemenschneider DE, Hall RB. Early rooting of dormant hardwood cuttings of Populus: analysis of quantitative genetics and genotype x environment interactions. Can J For Res Can Rech For. 2005;35:918-29.

39. Han K, Bradshaw HDJ, Gordon MP. Adventitious root and shoot regeneration in vitro is under major gene control in an F2 family of hybrid poplar (Populus trichocarpa * P. deltoides). For Genet. 1994;1 (3):139-46.

40. Schubert V, Weissleder A, Ali H, Fuchs J, Lermontova I, Meister A, Schubert I. Cohesin gene defects may impair sister chromatid alignment and genome stability in Arabidopsis thaliana. Chromosoma. 2009;118:591-605.

41. Zhao Y. Auxin biosynthesis: a simple two-step pathway converts tryptophan to indole-3-acetic acid in plants. Mol Plant. 2012;5:334-8.

42. Normanly J, Cohen JD, Fink GR. Arabidopsis thaliana auxotrophs reveal a tryptophan-independent biosynthetic pathway for indole-3-acetic acid. Proc Natl Acad Sci U S A. 1993;90:10355-9.

43. Wang B, Chu J, Yu T, Xu Q, Sun X, Yuan J, Xiong G, Wang G, Wang Y, Li J. Tryptophan-independent auxin biosynthesis contributes to early embryogenesis in Arabidopsis. Proc Natl Acad Sci. 2015;112:201503998.

44. Zeng ZB. Theoretical basis for separation of multiple linked gene effects in mapping quantitative trait loci. Proc Natl Acad Sci U S A. 1993;90:10972-6.

45. Churchill GA, Doerge RW. Empirical Threshold Values for Quantitative Trait Mapping. Genetics. 1994;138:963-71.

46. Chang S, Puryear J, Cairney J. A simple and efficient method for isolating RNA from pine trees. Plant Mol Biol Report. 1993;11:113-6.

47. Bolstad BM, Irizarry RA, Astrand M, Speed TP. A comparison of normalization methods for high density oligonucleotide array data based on variance and bias. Bioinformatics. 2003;19:185-93.

48. Storey JD, Tibshirani R. Statistical significance for genomewide studies. Proc Natl Acad Sci U S A. 2003;100:9440-5.

49. Higo K, Ugawa Y, Iwamoto M, Korenaga T. Plant cis-acting regulatory DNA elements (PLACE) database: 1999. Nucleic Acids Res. 1999;27:297-300.

\section{Submit your next manuscript to BioMed Central and we will help you at every step:}

- We accept pre-submission inquiries

- Our selector tool helps you to find the most relevant journal

- We provide round the clock customer support

- Convenient online submission

- Thorough peer review

- Inclusion in PubMed and all major indexing services

- Maximum visibility for your research

Submit your manuscript at www.biomedcentral.com/submit
() BioMed Central 